INPLASY

PROTOCOL

To cite: Zhao et al. Effect of electric stimulation on patients with facial paralysis: A systematic review and Metaanalysis of randomized controlled trials. Inplasy protocol 202210016. doi: 10.37766/inplasy2022.1.0016

Received: 05 January 2022

Published: 05 January 2022

Corresponding author: Jiangna Zhao

kfxszjn@163.com

Author Affiliation: Yueyang Hospital of Integrated Traditional Chinese and Western Medicine. Shanghai University of Traditional Chinese Medicine.

Support: SMC2013;81603713.

Review Stage at time of this submission: Preliminary searches.

Conflicts of interest:

None declared.

\section{Effect of electric stimulation on patients with facial paralysis: A systematic review and Meta-analysis of randomized controlled trials}

\author{
Zhao, J1; An, Y2; Yan, H3; Zhang, T4; Yan, J5.
}

Review question / Objective: To investigate the effectiveness of electric stimulation on patients with facial paralysis through a systematic review and meta-analysis.

Condition being studied: P: facial paralysis; I: electric stimulation; C: clinical routine treatment; $O$ : total clinical effectiveness, House-Brackmann scale (HBN) and Portmann scale; S:RCT.

Information sources: PubMed; the Cochrane Library; Embase; SinoMed; WanFang Data; Vip; China National Knowledge Infrastructure databases.

INPLASY registration number: This protocol was registered with the International Platform of Registered Systematic Review and Meta-Analysis Protocols (INPLASY) on 05 January 2022 and was last updated on 05 January 2022 (registration number INPLASY202210016).

\section{INTRODUCTION}

Review question / Objective: To investigate the effectiveness of electric stimulation on patients with facial paralysis through a systematic review and meta-analysis.
Condition being studied: P: facial paralysis; I: electric stimulation; C: clinical routine treatment; $O$ : total clinical effectiveness, House-Brackmann scale (HBN) and Portmann scale; S:RCT. 


\section{METHODS}

Participant or population: Who have been diagnosed with facial paralysis.

Intervention: Electric Stimulation.

Comparator: Clinical Routine Treatment.

Study designs to be included: RCT.

Eligibility criteria: Diagnostic criteria for facial paralysis in Neurology

Information sources: PubMed; the Cochrane Library; Embase; SinoMed; WanFang Data; Vip; China National Knowledge Infrastructure databases.

Main outcome(s): Total clinical effectiveness; House-Brackmann scale (HBN) ; Portmann scale.

Quality assessment / Risk of bias analysis: Cochrane Collaboration tools.

Strategy of data synthesis: Cochran's $\mathbf{Q}$ and 12 statistics were used to assess the heterogeneity between studies. This study defined $p>0.1$ and $12<50 \%$ as being of no significant heterogeneity, and fixed effects models were used for the meta-analysis. By applying sensitivity analysis to explore possible sources of Heterogeneity.

Subgroup analysis: Subgroup analysis was made according to course of disease, condition of disease, age, sex, intervention mode and so on.

Sensitivity analysis: These studies were removed one by one to examine the impact of individual studies on overall estimates and to assess the stability of pooled results.

Country(ies) involved: China.

Keywords: electric stimulation; facial paralysis; House-Brackmann scale; Portmann scale; meta-analysis.

Contributions of each author:

Author 1 - Jiangna Zhao.
Author 2 - Yun An.

Author 3 - Huixin Yan.

Author 4 - Tao Zhang.

Author 5 - Juntao Yan. 